\title{
Patient Activation Changes as a Potential Signal for Changes in Health Care Costs: Cohort Study of US High-Cost Patients
}

\author{
Ann Lindsay, M.D. ${ }^{7}$, Judith H. Hibbard, Dr.P. $H^{2}$, Derek B. Boothroyd, Ph. D ${ }^{3}$, Alan Glaseroff, M.D. ${ }^{4}$, and \\ Steven M. Asch, M.D. ${ }^{5}$ \\ 'Division of Primary Care and Population Health, Department of Medicine, Stanford University School of Medicine, McKinleyville, CA, USA; \\ ${ }^{2}$ University of Oregon, Eugene, USA; ${ }^{3}$ Quantitative Sciences Unit, Department of Medicine, Stanford University School of Medicine, Stanford, USA; \\ ${ }^{4}$ Center for Excellence in Clinical Research, Stanford University School of Medicine, Stanford, USA; ${ }^{5} V A$ Center for Innovation to Implementation, \\ Department of Medicine, Stanford University School of Medicine, Stanford, USA.
}

BACKGROUND: Programs to improve quality of care and lower costs for the highest utilizers of health services are proliferating, yet such programs have difficulty demonstrating cost savings.

OBJECTIVE: In this study, we explore the degree to which changes in Patient Activation Measure (PAM) levels predict health care costs among high-risk patients.

PARTICIPANTS: De-identified claims, demographic data, and serial PAM scores were analyzed on 2155 patients from multiple medical groups engaged in an existing Center for Medicare and Medicaid Innovation-funded intervention over 3 years designed to activate and improve care coordination for high-risk patients.

DESIGN: In this prospective cohort study, four levels of PAM (from low to high) were used as the main predictor variable. We fit mixed linear models for $\log _{10}$ of allowed charges in follow-up periods in relation to change in PAM, controlling for baseline PAM, baseline costs, age, sex, income, and baseline risk score.

MAIN MEASURES: Total allowed charges were derived from claims data for the cohort. PAM scores were from a separate database managed by the local practices.

KEY RESULTS: A single PAM level increase was associated with $8.3 \%$ lower follow-up costs (95\% confidence interval 2.5-13.2\%).

CONCLUSIONS: These findings contribute to a growing evidence base that the change in PAM score could serve as an early signal indicating the impact of interventions designed for high-cost, high-needs patients.

KEY WORDS: medicare; patient activation; health economics; return on investment.

J Gen Intern Med 33(12):2106-12

DOI: $10.1007 / \mathrm{s} 11606-018-4657-6$

(c) Society of General Internal Medicine 2018

Electronic supplementary material The online version of this article (https://doi.org/10.1007/s11606-018-4657-6) contains supplementary material, which is available to authorized users.

Received January 11, 2018

Revised June 29, 2018

Accepted August 17, 2018

Published online October 5, 2018

\section{INTRODUCTION}

Restraining costs and improving quality of care have become an urgent priority. ${ }^{1}$ Unfortunately, programs focusing on highrisk high-cost populations have difficulty demonstrating cost savings, making it hard for subsequent programs to learn from their efforts. Randomized trials are challenging to conduct and are often insufficiently powered to produce significant results. Claims data are generally not available for 6 months or more after service delivery and programs might serve patients in multiple health insurance plans, making aggregating total cost data difficult. Furthermore, complete claims data may not be available for analysis, much less for a propensity-matched control group, and a few high-cost outliers often skew the results. Finally, pre-post studies may reflect regression to the mean. ${ }^{2}$ Therefore, it would be advantageous to have an early indicator or a proxy for changes in costs to allow an estimate of the relative effectiveness of different interventions, even when numbers are small and claims analysis unavailable or delayed.

In this analysis, we explore whether changes in the Patient Activation Measure (PAM) score could serve as a potential proxy or an early indicator for changes in cost in a high-cost US Medicare population. The PAM is a 13-item survey assessing an individual's knowledge, skill, and confidence for self-management. ${ }^{3}$ A low score indicates that patients are less likely to engage in effective self-management. The PAM score is broken down to four levels. At level one, patients may not understand the need to take an active role in their own health. At level two, they may still lack confidence and skill to take action. At PAM, level three patients are beginning to take action. At level four, patients may have difficulty maintaining positive health behaviors over time. The PAM survey is used in clinical settings to better support patient self-management and is easy to administer. ${ }^{4}$ Scores can be entered into a database and tracked over time.

Research indicates that less activated patients are less knowledgeable about their conditions, less adherent to treatment regimens, less likely to communicate concerns to providers, and less likely to recognize serious symptoms or know how to handle these symptoms when they arise. ${ }^{5-7}$ Prospective studies indicate that, after controlling for demographics 
and chronic illness status, less activated patients are more likely to be hospitalized and to use the emergency room (ED) than are higher activated patients. ${ }^{8,} 9$ These differences have also been observed within a high-risk population. ${ }^{9}$

While there is significant evidence that PAM correlates with costs, only some of the evidence is based on longitudinal data and that evidence, mostly confined to a single health insurance plan or institution, is not focused on high utilizers or tested in an interventional context. ${ }^{8-11}$

We leverage an existing intervention specifically designed to activate patients, the Intensive Outpatient Care Program, to test the relationship between change in PAM and subsequent costs. We focus on this high-cost patient cohort from multiple primary care practices in different systems from across a wide geographic region in the western USA.

The Center for Medicare and Medicaid Innovation funded Pacific Business Group on Health to implement and test efficacy of an Intensive Outpatient Care Program (IOCP). PBGH led 23 medical groups in California, Nevada, Arizona, Idaho, and Washington in a pilot to manage medically complex Medicare recipients at risk for high costs of care. Patients were enrolled from January 1, 2014 to July 31, 2015. Each patient was assigned a care coordinator, licensed or nonlicensed staff with specific training in motivational interviewing, who was embedded in the primary care team to develop relationships with patients and foster delivery of highly individualized and accessible primary care. Less activated patients with lower PAM scores received more support. Patients' action plans were based on individual treatment goals and tailored to their activation level, encouraging plans realistic for success. As health outcomes and costs for this population are determined to a significant degree by the patient's ability and willingness to self-manage, ${ }^{9}$ requiring knowledge, skill, and confidence and reflected in their patient activation scores, improving patients' activation scores was identified as an explicit intermediate outcome in the IOCP. The interventional group experienced a $21 \%$ decrease in overall costs. ${ }^{12}$ We use data from patients in the IOCP intervention group and assess the degree to which increases in activation scores are predictive of decreases in overall costs of care in the immediate post intervention period.

We seek to provide added evidence and credibility for the use of PAM as a proxy for future cost savings.

We hypothesize that when PAM level improves, costs will go down, and that when PAM level declines, costs will increase.

\section{METHODS}

This is a prospective cohort study. The cohort are all patients from the IOCCP demonstration project. The IOCP recruited "high risk" patients based on potential for incurring future high health care costs. Most patients were recruited based on their prospective risk [using Milliman's Advanced Risk
Adjuster (MARA) score], plus the clinical judgment of their provider of the likelihood of imminent adverse outcomes. Other patients were recruited following emergency department and acute care admissions. All patients were covered by Medicare, either in managed care or in fee for service arrangements.

To be included in this analysis, patients must have had a PAM score performed at baseline and a follow-up score 4 to 12 months later, plus a complete claims history for 6 months pre- and 6 months post enrollment. (For patients with more than one PAM score between 4 and 12 months after baseline, the one closest to 6 months was used.)

The PAM score was obtained at enrollment and approximately every 4-12 months. The PAM score was part of a separate database managed by the local practices and aggregated by the IOCP program. De-identified demographic data, including average income based on zip code and total cost of care based on allowed Medicare charges, were derived from claims data for 6 months prior to the intervention and for the 6 or 12 months following enrollment (depending on length of available claims follow-up). The cost of the IOCP program itself was not included in the analysis. As part of the data deidentification process, age was provided to the study team in 14 categories and income was provided in 31 categories. Categories were converted to numeric values using the midpoints of the intervals except for age $<65$, age $\geq 90$, and income $\geq \$ 100,000$ for which we assigned values of 60,92 , and $\$ 105,000$ (only one patient in this category in our analytic population).

PAM is scored on a $0-100$ scale, which can be broken down into four levels of activation (from low to high). We used the four levels of PAM as our main predictor variable ${ }^{3}$ when modeling follow-up allowed total charges. To assess change in PAM, we used the difference between the follow-up PAM level and the baseline PAM level, ranging from -3 for a decrease of 3 to +3 for an increase of 3 PAM levels.

The patients with adequate PAM and allowed charges data were compared to the other patients using Fisher's exact test for age and Wilcoxon rank sum tests for other baseline variables. We tested for differences in unadjusted observed costs in the first 6 months of follow-up in different patient subgroups with Kruskal-Wallis tests.

For our primary analyses, we added the total allowed charges for all claims in the time periods. We fit mixed linear models for the base 10 logarithm $(\log 10)$ of allowed charges in the two follow-up periods that included a random effect for individual (to account for correlation between the two periods) and fixed effects for the following a priori set of predictors: $\log 10$ of baseline allowed charges, sex, numeric versions of income (estimated based on zip codes) and age, and the two age indicators, $\log 10$ of the MARA risk score, the follow-up period, the baseline PAM level, the change in PAM level between the baseline PAM and the follow-up PAM, and an interaction between follow-up period and the change in PAM level. (The logarithmic transformations were used to account 
for the highly skewed nature of the cost and MARA variables.)

(See Online Appendix A for calculation detail.)

The mixed linear models were fit by restricted maximum likelihood with bootstrap $p$ values and percentile bootstrap confidence intervals calculated for model parameters using 1000 bootstrap replicates. To convert the models for $\log 10$ of charges back to the scale of interest (\$), we used a "smearing" approach to account for the non-linearity of the transformations. ${ }^{13}$ (For each model estimate on the log scale, a set of numbers was created by adding each of the residuals from the model to the estimate. These were then transformed back to the cost scale by calculating 10 to the power of each of those numbers and the final estimate was the mean of those transformed values.) When calculating estimates for a combination of baseline PAM level and change in PAM level (for Table 3 and Fig. 1), we used only those patients with that combination to account for the differences in other covariates by PAM level.

All statistical analyses were done using $\mathrm{R}$ version 3.1.1. ${ }^{14}$ Mixed linear models were fit using R package lmerTest. ${ }^{15}$

\section{RESULTS}

There were 4617 patients with complete claims history (6 months pre- and at least 6 months post enrollment). Of the 4617 patients in the study cohort, 2329 had fewer than 2 PAM scores during the study period. Eighty-nine percent of patients had at least an initial PAM assessment completed. Patients in the program less than 6 months did not have follow-up assessments done. One hundred twenty-three of those with 2 scores did not have a score in the window from 4 to 12 months after baseline, and 10 had a first PAM after June 30, 2014. This left 2155 patients with at least 2 PAM scores for analyses collected at approximately 6-month intervals during the study period. The median time to the second PAM was 6.3 months, interquartile range 5.7-6.8 months.

The 2155 patient study cohort are on average 2 years younger and have higher income and lower risk score than the larger population of participants without two PAM scores in the specified time frame. (That is to say, eligible participants were slightly more advantaged in terms of age, income, and health status, as compared to non-participants. See Online Appendix B.)

During the intervention, $35 \%$ of patients increased a full PAM level or more.

Table 1 shows the bivariate relationship between baseline study variables and costs in the first 6 months of follow-up. Post intervention costs are significantly associated with baseline costs, risk scores, and inversely related to PAM level. Demographics are not significantly linked with post intervention costs. Small numbers of patients had missing values for sex, income, and MARA score and only complete case analyses were used for linear models.

Table 2 shows the multivariate relationship between PAM level change and total costs. After controlling for baseline

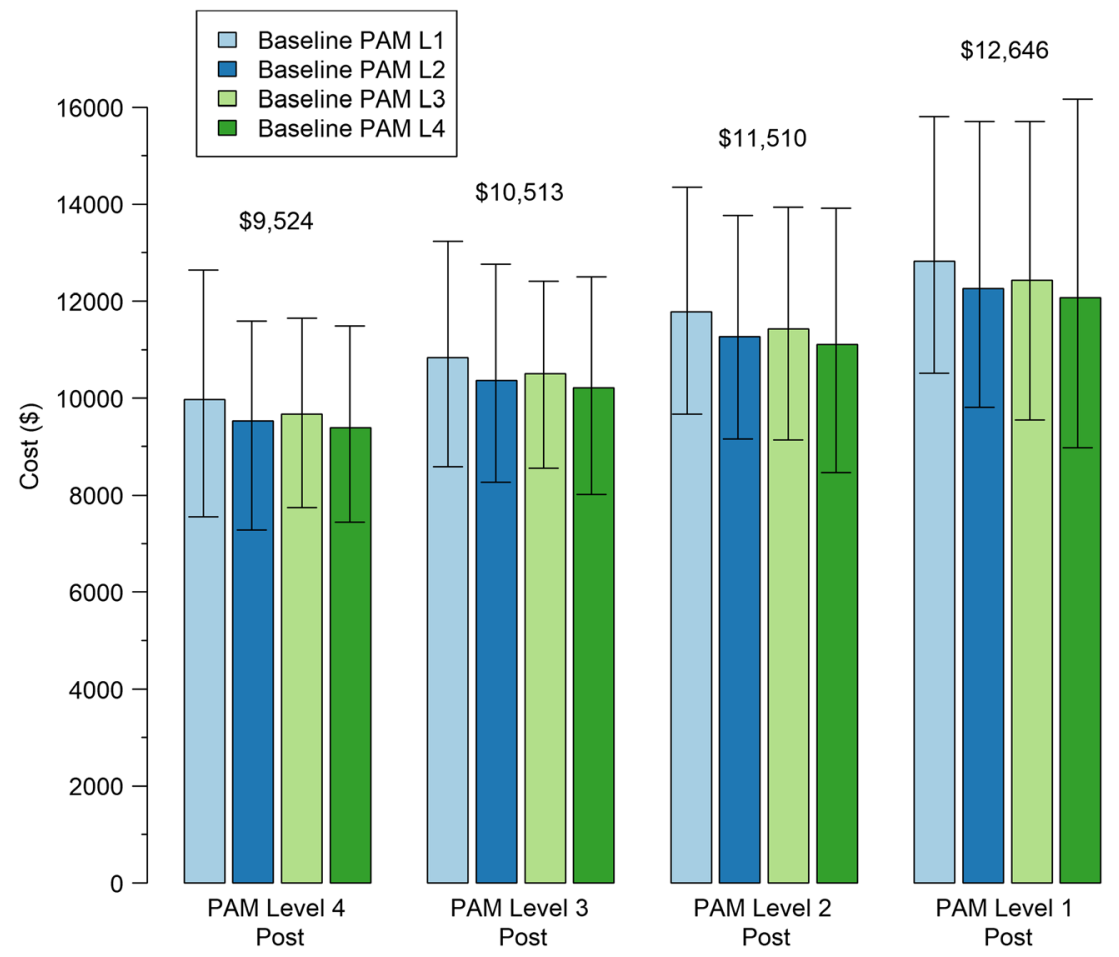

Fig. 1 Predicted costs based on baseline PAM level and post PAM level. Controlling for baseline costs, sex, age income, and baseline risk score. Numerical values above each set of 4 bars are the weighted average of the values of those bars, weighted by the relative prevalence of the combinations of baseline PAM, and follow-up PAM in the cohort. Estimates are highly correlated so the widths of the bootstrap confidence intervals do not reflect relative differences. 
Table 1 Bivariate Relationship Between Total Follow-up Costs in the First 6 Months and Key Baseline Study Variables for the 2155 Analyzed Patients

\begin{tabular}{|c|c|c|c|c|c|}
\hline & & $N$ & Median & IQR & $p$ value \\
\hline \multirow[t]{2}{*}{ Sex } & Male & 878 & $\$ 3249$ & $(\$ 1314, \$ 8947)$ & 0.10 \\
\hline & Female & 1269 & $\$ 2842$ & $(\$ 1302, \$ 7058)$ & \\
\hline \multirow{4}{*}{ Age } & Up to 70 & 355 & $\$ 3368$ & $(\$ 1177, \$ 9137)$ & 0.77 \\
\hline & 71 to 80 & 609 & $\$ 3027$ & $(\$ 1394, \$ 7295)$ & \\
\hline & 81 to 89 & 604 & $\$ 2861$ & $(\$ 1305, \$ 7162)$ & \\
\hline & 90 and over & 587 & $\$ 2917$ & $(\$ 1241, \$ 7552)$ & \\
\hline \multirow{4}{*}{ Income } & Under $\$ 30,000$ & 425 & $\$ 2917$ & $(\$ 1277, \$ 7424)$ & 0.27 \\
\hline & $\$ 30 \mathrm{~K}$ to $\$ 50 \mathrm{~K}$ & 840 & $\$ 2748$ & $(\$ 1302, \$ 7062)$ & \\
\hline & $\$ 50 \mathrm{~K}$ to $\$ 70 \mathrm{~K}$ & 539 & $\$ 3279$ & $(\$ 1421, \$ 8127)$ & \\
\hline & $\$ 70 \mathrm{~K}$ and over & 286 & $\$ 2863$ & $(\$ 1182, \$ 7742)$ & \\
\hline \multirow[t]{4}{*}{ Baseline 6-month costs } & Under $\$ 2500$ & 567 & $\$ 1441$ & $(\$ 639, \$ 3404)$ & $<0.001$ \\
\hline & $\$ 2.5 \mathrm{~K}$ to $\$ 6 \mathrm{~K}$ & 486 & $\$ 2799$ & $(\$ 1537, \$ 5187)$ & \\
\hline & $\$ 6 \mathrm{~K}$ to $\$ 15 \mathrm{~K}$ & 483 & $\$ 4045$ & $(\$ 1814, \$ 9140)$ & \\
\hline & $\$ 15 \mathrm{~K}$ and over & 619 & $\$ 5346$ & $(\$ 2080, \$ 17,880)$ & \\
\hline \multirow{4}{*}{ MARA Risk Score } & Under 1.5 & 455 & $\$ 1614$ & $(\$ 537, \$ 4492)$ & $<0.001$ \\
\hline & 1.5 to 3 & 643 & $\$ 1839$ & $(\$ 909, \$ 3381)$ & \\
\hline & 3 to 5 & 470 & $\$ 3398$ & $(\$ 1810, \$ 7333)$ & \\
\hline & 5 and over & 542 & $\$ 7468$ & $(\$ 3575, \$ 22,764)$ & \\
\hline \multirow[t]{4}{*}{ Baseline PAM level } & 1 & 552 & $\$ 3410$ & $(\$ 1387, \$ 9948)$ & 0.050 \\
\hline & 2 & 403 & $\$ 2888$ & $(\$ 1284, \$ 7156)$ & \\
\hline & 3 & 842 & $\$ 2978$ & $(\$ 1318, \$ 7558)$ & \\
\hline & 4 & 358 & $\$ 2711$ & $(\$ 1223, \$ 5866)$ & \\
\hline
\end{tabular}

Missing counts: Sex, 8; Income, 65; MARA score, 45. IQR is interquartile range (25th percentile, 75th percentile). The p values are from Kruskal-Wallis tests

PAM, baseline costs, age, sex, income, and baseline risk score, change in PAM level change is a significant independent predictor of costs in the post intervention period with an estimate of $8.3 \%$ lower costs (95\% CI 2.5-13.2\%) per one increase in PAM level. (We included an interaction term to allow for different effects in the first and second 6 months of follow-up but that term was negligible and not statistically significant.) Baseline PAM is also a significant predictor, with an estimate of $6.3 \%$ lower costs (95\% CI 1.7 to $10.7 \%$ lower) per PAM level.

Cost differentials observed by PAM level change appear to be largely a result of changes in emergency department use and hospital admissions (analysis not shown).
Table 3 shows adjusted costs in the post period by PAM level change. Costs are adjusted for age, sex, income, baseline costs, and baseline risk score. When patients improve one PAM level, it appears to reduce costs $8 \%$, regardless of the baseline PAM level. Among those who improved two PAM levels, a $15 \%$ reduction in costs was observed. Declining PAM levels yielded slightly greater impacts, with costs rising 9 and $18 \%$ for PAM level changes of 1 or 2 levels respectively.

Figure 1 provides another view into the relationship between PAM level changes and cost outcomes. We examined total allowed charges for patients who became highly activated or stayed highly activated versus those who stayed less activated or became less activated. For example, those at PAM

Table 2 PAM Level Change as a Predictor of Costs in the Post Intervention Period: A Linear Regression Model. Models are for total cost (not restricted to hospital or ED)

\begin{tabular}{|c|c|c|c|c|}
\hline \multicolumn{5}{|l|}{ Primary model } \\
\hline & Estimate & Std. error & $\begin{array}{l}\text { Multiplicative factor } \\
\text { (Bootstrap 95\% CI) }\end{array}$ & Bootstrap $p$ value \\
\hline Intercept & 2.897 & 0.190 & & \\
\hline Baseline PAM level & -0.027 & 0.014 & $0.94(0.89,0.98)$ & 0.004 \\
\hline Change in PAM level & -0.038 & 0.016 & $0.92(0.87,0.98)$ & 0.006 \\
\hline FU.period $=$ months 7 to 12 & -0.175 & 0.022 & $0.67(0.55,0.70)$ & $<0.001$ \\
\hline Interaction (change in PAM level $*$ FU.period $=$ months 7 to 12 ) & 0.003 & 0.021 & $1.01(0.88,1.11)$ & 0.79 \\
\hline $\log 10$ of charges in 6 months before intervention & 0.194 & 0.017 & $1.56(1.47,1.67)$ & $<0.001$ \\
\hline Male sex & 0.017 & 0.024 & $1.04(0.96,1.13)$ & 0.37 \\
\hline Approximate age & -0.005 & 0.002 & $0.99(0.98,1.00)$ & 0.002 \\
\hline Age $<65$ & -0.010 & 0.066 & $0.98(0.78,1.23)$ & 0.88 \\
\hline Age $\geq 90$ & 0.055 & 0.040 & $1.13(1.00,1.28)$ & 0.064 \\
\hline Approximate income (per $\$ 10,000$ ) & 0.003 & 0.006 & $1.01(0.99,1.03)$ & 0.59 \\
\hline log10 of baseline MARA risk score & 0.661 & 0.034 & $4.59(4.10,5.17)$ & $<0.001$ \\
\hline
\end{tabular}

A linear model on a logarithmic scale becomes a multiplicative model on the untransformed values. The multiplicative factor is the factor by which follow-up cost is estimated to be multiplied for either a 1 unit increase in a numeric predictor or for a yes value. Hence, e.g., follow-up cost is estimated to be multiplied by 0.917 , i.e., decreased by $8.3 \%$ for each increase in PAM level 
Table 3 Adjusted Average Costs when PAM Level Changes

\begin{tabular}{|c|c|c|c|}
\hline \multicolumn{3}{|c|}{ Translation of coefficients into adjusted average costs per patient } & \multirow{2}{*}{$\begin{array}{l}\text { Percent change in costs when } \\
\text { PAM level changes } \\
\text { Percent change in adjusted costs when } \\
\text { PAM level changes (as compared to } \\
\text { staying at the same level) }\end{array}$} \\
\hline $\begin{array}{l}\text { PAM level baseline and changes } \\
\text { in the post intervention period }\end{array}$ & $\begin{array}{l}\text { Adjusted costs when PAM } \\
\text { level remains the same from } \\
\text { pre to post intervention period }\end{array}$ & $\begin{array}{l}\text { Adjusted costs when PAM } \\
\text { level changes from pre to } \\
\text { post intervention }\end{array}$ & \\
\hline \multicolumn{4}{|c|}{ When PAM level goes up one level in post period } \\
\hline Baseline level 1 & $\$ 12,068$ & $\$ 11,099$ & Costs down $8 \%$ \\
\hline Baseline level 2 & $\$ 11,332$ & $\$ 10,433$ & Costs down $8 \%$ \\
\hline Baseline level 3 & $\$ 10,641$ & $\$ 9786$ & Costs down $8 \%$ \\
\hline \multicolumn{4}{|c|}{ When PAM goes up two levels at post } \\
\hline Baseline level 1 & $\$ 12,068$ & $\$ 10,207$ & Costs down $15 \%$ \\
\hline Baseline level 2 & $\$ 11,332$ & $\$ 9585$ & Costs down $15 \%$ \\
\hline \multicolumn{4}{|c|}{ When PAM goes up three levels at post } \\
\hline Baseline level 1 & $\$ 12,068$ & $\$ 9388$ & Costs down $22 \%$ \\
\hline \multicolumn{4}{|c|}{ When PAM goes down one level at post } \\
\hline Baseline level 2 & $\$ 11,332$ & $\$ 12,322$ & Costs up $9 \%$ \\
\hline Baseline level 3 & $\$ 10,641$ & $\$ 11,571$ & Costs up $9 \%$ \\
\hline Baseline level 4 & $\$ 9992$ & $\$ 10,865$ & Costs up $9 \%$ \\
\hline \multicolumn{4}{|c|}{ When PAM goes down two levels at post } \\
\hline Baseline level 3 & $\$ 10,641$ & $\$ 12,582$ & Costs up $18 \%$ \\
\hline Baseline level 4 & $\$ 9992$ & $\$ 11,814$ & Costs up $18 \%$ \\
\hline \multicolumn{4}{|c|}{ When PAM goes down three levels at post } \\
\hline Baseline level 4 & $\$ 9992$ & $\$ 12,847$ & Costs up $29 \%$ \\
\hline
\end{tabular}

Costs are adjusted by baseline costs, sex, age, income, and baseline risk score

level 4 1-year later, regardless of baseline level, had cost estimates between $\$ 9388$ and $\$ 9786$, a relatively narrow cost band. Those who started at PAM level one and rose to level 4 had costs very similar to those who stayed at PAM level 4. Those who ended up at PAM level 1, regardless of their baseline PAM level, had costs 1 year after their baseline PAM score between $\$ 12,068$ and $\$ 12,847$, also a relatively narrow band. Using weighted average costs for each of the groups weighted by the number of patients in the groups in our cohort shows that there is a $33 \%$ differential in costs for those who went to a high level of activation or stayed at a high activation level compared to those who declined to the lowest level of activation or stayed at the lowest level of activation.

\section{DISCUSSION}

We found an increase in activation level was associated with subsequent costs decreases in a high-risk population. This association persisted when controlling for common predictors of costs including sex, age, income, and comorbidities. These findings support the hypothesis that improved activation may be a useful intermediate marker for programs designed to control costs of care for high utilizers. The findings are similar to those of another cohort study. ${ }^{16}$ In that study, those staying at the lower levels of activation over a 2 -year period had costs that were $31 \%$ higher (compared to $33 \%$ higher in the current study) than those remaining at the highest level of activation. The current study findings go further and highlight that the follow-up activation levels are predictive of outcome costs, regardless of baseline PAM level.

These results contribute to a growing evidence base that the PAM score is associated with health behaviors, clinical outcomes, and the costs of care..$^{9,11,17-19}$ It appears that activation affects outcomes by operating through patient behaviors. For example, less activated patients, because of poor self-management practices, are more likely to have avoidable emergency department visits and hospital stays than are higher activated patients. ${ }^{11}$ Regardless of MARA risk level, changes in PAM scores over time have been shown to be linked with cost changes in the expected direction. ${ }^{20}$ Targeted efforts, like the one described here, have been shown to increase patient activation. $^{21,22}$

Several aspects of the current study are novel. First, a limited number of previous studies evaluated the longitudinal relationship between activation and cost. $8,23,24$ The observed longitudinal relationships of this study make conclusions more robust than previous cross-sectional observations. Second, many previous studies were confined to single institutions, while the current study included 23 different medical groups in five states. Third, our work was conducted in the context of an intervention, while many previous studies observed only natural relationships. Because the intervention improved PAM scores, and improved PAM scores were associated with reduced costs, the potential role of PAM as an intermediate outcome metric is supported.

There are limitations to these conclusions. Our analysis is confined to a population residing in the USA, covered by Medicare, and receiving the intervention. Yet, as the intervention was intended to increase activation and was associated with increased activation, it is reasonable to hypothesize the association between the intervention, activation, and subsequent costs. We were only able to control for a small set of clinical and demographic variables: unobserved confounding variables could explain some of our findings. In addition, only half the participants had a second PAM score, limiting the 
analysis to only those with two scores, and potentially reducing the generalizability of the findings. Further testing with different populations would help to assess the validity and generalizability of the findings.

Regression to the mean is a common confounder in studies assessing health care costs. When costs decline following an intervention, it is difficult to determine whether those declines are a result of the intervention or the regression to the mean in costs that would have occurred regardless of the intervention. In this analysis, however, we see that costs decline when PAM levels increase, and they increase when PAM levels decline, providing some evidence that regression to the mean does not explain the results observed in this study.

\section{CONCLUSIONS}

The implications for clinical leaders and policymakers are profound. Patient activation measures may provide an early signal of success or failure for interventions designed to care for the highest utilizers. These interventions have spread rapidly as financial incentives swing away from pure fee-forservice reimbursement. Parsing out what program components best activate patients can guide their development. In this intervention, the patients' care plans were tailored to their level of activation, encouraging patient behaviors realistic for them to be successful with. In addition, the amount of support the patient received was PAM-tailored, providing more support to the less activated patients. Other studies have shown tailoring coaching and care plans to the patient's activation level are effective in increasing activation. ${ }^{21,22,25}$

Patient behavior, which includes ability to effectively selfmanage, has been shown to be more important to outcomes than the provision of medical care. ${ }^{26}$ Patient activation may provide the link between those behaviors and interventions designed to foster them. Moreover, as the evidence mounts that activation predicts subsequent costs, the business case for developing "activation" programs builds.

The findings indicate that post intervention PAM level is predictive of costs, regardless of the pre-intervention PAM level, providing added support to using PAM as a proxy for costs. Using PAM level change as a proxy for costs would allow those testing delivery system innovations early feedback on the impact of interventions, especially helpful where timely and complete cost data is not available. Easier prediction of potential savings could facilitate projections of return on investment for programs for which the cost of the intervention is known.

Corresponding Author: Ann Lindsay, M.D.; Division of Primary Care and Population Health, Department of Medicine Stanford University School of Medicine, 2475 North Bank Rd., McKinleyville, CA 95519, USA (e-mail: ann.lindsay@stanford.edu).

Funding Information Funding for the analysis reported in this article was provided from the Stanford Coordinated Care Team Training Account at the Stanford School of Medicine.

\section{Compliance with Ethical Standards:}

Conflict of Interest: Ann D. Lindsay is a clinical advisor of Clover Health.

Judith $H$. Hibbard is a consultant and equity stakeholder for Insignia Health.

Alan Glaseroff is a clinical advisor for Cardinal Analytx, VIM, Omada Health, and Clover Health.

All remaining authors declare that they do not have a conflict of interest.

\section{REFERENCES}

1. Blumenthal D, Chernof B, Fulmer T, Lumpkin J, Selberg J. Caring for High-Need, High-Cost Patients - An Urgent Priority. $N$ Engl J Med. 2016;375(10):909-911. doi:https://doi.org/10.1056/NEJMp1608511.

2. Zulman DM, Pal Chee C, Ezeji-Okoye SC, et al. Effect of an Intensive Outpatient Program to Augment Primary Care for High-Need Veterans Affairs Patients. JAMA Intern Med. 2016;5(4):e007771. doi:https://doi. org/10.1001/jamainternmed.2016.8021.

3. Hibbard JH, Mahoney ER, Stockard J, Tusler M. Development and testing of a short form of the Patient Activation Measure. Heal Serv Res. 2005;40. doi:https://doi.org/10.1111/j.1475-6773.2005.00438.x.

4. Hibbard JH, Stockard J, Mahoney ER, Tusler M. Development of the Patient Activation Measure (PAM): conceptualizing and measuring activation in patients and consumers. Health Serv Res. 2004;39(4 Pt 1):1005-1026. doi:https://doi.org/10.1111/j.1475-6773.2004.00269.x.

5. Evangelista LS, Liao S, Motie M, De Michelis N, Lombardo D. On-going palliative care enhances perceived control and patient activation and reduces symptom distress in patients with symptomatic heart failure: a pilot study. Eur J Cardiovasc Nurs. 2014;13(2):116-123. doi:https://doi. org/10.1177/1474515114520766.

6. Salgado TM, Mackler E, Severson JA, et al. The relationship between patient activation, confidence to self-manage side effects, and adherence to oral oncolytics: a pilot study with Michigan oncology practices. Support Care Cancer. 2017:1-11. doi:https://doi.org/10.1007/s00520-0173584-0.

7. Hibbard JH, Mahoney E, Sonet E. Does Patient Activation Level Affect the Cancer Patient Journey? Patient Educ Couns. 2017. doi:https://doi.org/ 10.1016/j.pec.2017.03.019.

8. GreeneJ, Hibbard JH, Sacks R, Overton V, Parrotta CD. When patientactivation levels change, health outcomes and costs change, too. Health Aff (Millwood). 2015;34(3):431-437. doi:https://doi.org/10.1377/hlthaff.2014.0452.

9. Hibbard JH, Greene J, Sacks R, Overton V, Parrotta CD. Adding A Measure Of Patient Self-Management Capability To Risk Assessment Can Improve Prediction Of High Costs. Health Aff. 2016;35(3):489-494. doi:https://doi.org/10.1377/hlthaff.2015.1031.

10. Hibbard JH, Greene J, Overton V. Patients with lower activation associated with higher costs; delivery systems should know their patients' "scores". Health Aff. 2013;32(2):216-222. doi:https://doi.org/ 10.1377/hlthaff.2012.1064.

11. Hibbard JH, Greene J, Sacks RM, Overton V, Parrotta C. Improving Population Health Management Strategies: Identifying Patients Who Are More Likely to Be Users of Avoidable Costly Care and Those More Likely to Develop a New Chronic Disease. Health Serv Res. 2016. doi:https://doi. org/10.1111/1475-6773.12545.

12. Pantely, SE; Lee C. PBGH IOCP Experience Summary. The project described was supported by Grant Number 1C1CMS331047 from the Department of Health and Human Services, Centers for Medicare \& Medicaid Services. The contents of this publication are solely the responsibility of the authors and do not necessarily represent the official views of the U.S. Department of Health and Human Services or any of its agencies. The research presented here was conducted by the awardee. Findings might or might not be consistent with or confirmed by the findings of the independent evaluation contractor. The data on which this study is based was shared with the researchers for a limited time by agreements between the Centers for Medicare and Medicaid Services, the Pacific Business Group on Health (awardee of the CMS grant) and Stanford School of Medicine. The data will not be available to other researchers.

13. Duan N. Smearing Estimate: A Nonparametric Retransformation Method J Am Stat Assoc. 1983;78(383):605-610. doi:https://doi.org/10.1080/ 01621459.1983.10478017. 
14. R. Core Team. R: The R Project for Statistical Computing.

15. Kuznetsova A. Tests in Linear Mixed Effects Models [R package lmerTest version 2.0-33].

16. Greene J, Hibbard JH, Sacks R, Overton V, Parrotta CD. When Patient Activation Levels Change, Health Outcomes And Costs Change, Too. Health Aff. 2015;34(3):431-437. doi:https://doi.org/10.1377/hlthaff. 2014.0452 .

17. Hibbard JH, Greene J, Overton V. Patients with lower activation associated with higher costs; Delivery systems should know their Patients' "Scores". Health Aff. 2013;32:216-222. doi:https://doi.org/ 10.1377/hlthaff.2012.1133.

18. Fowles JB, Terry P, Xi M, Hibbard J, Bloom CT, Harvey L. Measuring selfmanagement of patients' and employees' health: Further validation of the Patient Activation Measure (PAM) based on its relation to employee characteristics. Patient Educ Couns. 2009;77:116-122. http://www. sciencedirect.com/science/article/pii/S0738399109000949.

19. Remmers C, Hibbard J, Mosen DM, Wagenfield M, Hoye RE, Jones C. Is Patient Activation Associated With Future Health Outcomes and Healthcare Utilization Among Patients With Diabetes ? J Ambul Care Manag. 2009;32(4): 1-8.

20. Greene J, Hibbard JH, Sacks R, Overton V, Parrotta CD. When patient activation levels change, health outcomes and costs change, too. Health
Aff. 2015;34(3):431-437. doi:https://doi.org/10.1377/hlthaff.2014. 0452.

21. Hibbard JH, Greene J, Tusler M. Improving the outcomes of disease management by tailoring care to the patient's level of activation. Am J Manag Care. 2009; 15(6):353-360. .

22. Shively MJ, Gardetto NJ, Kodiath MF, et al. Effect of patient activation on self-management in patients with heart failure. $J$ Cardiovasc Nurs. 2013;28:20-34. doi:https://doi.org/10.1097/JCN.0b013e318239f9f9.

23. Mercer SW, Jani BD, Maxwell M, Wong SYS, Watt GCM. Patient enablement requires physician empathy: a cross-sectional study of general practice consultations in areas of high and low socioeconomic deprivation in Scotland. BMC Fam Pract. 2012;13(1):6. doi:https://doi. org/10.1186/1471-2296-13-6.

24. Hibbard JH, Greene J, Shi Y, Mittler J, Scanlon D. Taking the Long View: How Well Do Patient Activation Scores Predict Outcomes Four Years Later? Med Care Res Rev. 2015;72(3):324. doi:https://doi.org/10.1177/ 1077558715573871.

25. Reistroffer, Cindy; Hearld, Larry; and Szychowski J. An Examination of the Relationship Between Care Management With Coaching for Activation and Patient Outcomes. Am J Manag Care. 2017;23(2):123-128.

26. Schoeder S. We Can Do Better - Improving the Health of the American People - NEJM. N Engl J Med. 2007. 